\title{
Sodium channel blocker protects axons in MS models
}

A drug that is currently in phase III trials for the treatment of Parkinson disease might also protect against axonal degeneration in multiple sclerosis (MS), according to new research published in Brain. Damineh Morsali and colleagues found that the sodium channel blocker safinamide preserved axonal integrity and function in two rat models of MS.

"Currently approved immunomodulatory therapies in MS mainly target the acquired immune system outside the CNS, but accumulating evidence implies that axonal degeneration and loss of function may be due to activation of innate immune cells within the CNS and an energy deficit within the axons," explains Morsali.

The researchers induced experimental autoimmune encephalomyelitis (EAE) - a condition that closely resembles MS-in rats via inoculation with spinal cord homogenate or recombinant myelin oligodendrocyte glycoprotein. In both EAE models, rats treated with the higher dose of safinamide for 2 weeks showed greater numbers of surviving and functional axons than did controls treated with saline. In addition, only $10 \%$ of safinamidetreated rats exhibited bilateral hindlimb paralysis at the end of the trial, compared with $65 \%$ of controls.

"Safinamide provided significant protection of both function and structure even if its administration was delayed until after the onset of neurological deficits-a strategy that is clinically especially relevant," says Morsali.

The results of further in vivo and cell culture studies were consistent with a dual mechanism of action of safinamide in EAE: alleviation of a neuronal energy deficit, and reduction of microglial activation. The drug increased production of the antioxidant glutathione in microglial cultures, suggesting a switch to an anti-inflammatory phenotype in these cells.

Another sodium channel blocker, lamotrigine, has previously shown some promise in patients with secondary progressive MS. Owing to tolerability concerns, however, this drug has not so far been pursued further in the MS context. "If safinamide proves to be better tolerated, it would be appropriate to consider a clinical trial of safinamide for neuroprotection in MS," suggests Morsali.

Heather Wood

Original article Morsali, D. et al. Safinamide and flecainide protect axons and reduce microglial activation in models of multiple sclerosis. Brain 136, 1067-1082 (2013) 